\title{
Veno-arterial extracorporeal membrane oxygenation as a bridge to recovery in acute aluminium phosphide poisoning: A case report
}

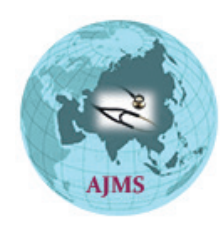

\author{
Arvind Kumar', Jai Prakash², Gaurav Arya², Anand Kumar Yadav³, Aman Dhankhar ${ }^{4}$ \\ ${ }^{1}$ Director Critical Care Services, ${ }^{2}$ Consultant, Department of Anesthesiology and Critical Care Services, ${ }^{3}$ Consultant \\ Surgeon, ${ }^{4}$ Resident Medical Officer Emergency Medicine, Kainos Super-Speciality Hospital, Rohtak, Haryana, India
}

\section{A B S T R A C T}

Aluminium phosphide (AIP) is a common fumigant used for controlling rodents and pests in grain storage facilities. Its consumption is reported in accidental or suicidal cases carrying high mortality. In the present case, a young male was reported with ingestion of AIP with severe lactic acidosis and systolic dysfunction. The patient was managed with extracorporeal membrane oxygenation. In reserved cases, ECMO can be instituted taking in view of risk and complications.

Key words: Aluminium phosphide; Phosphine; Extracorporeal membrane oxygenation; Ventilator; Acidosis; Systolic dysfunction

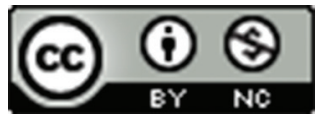

This work is licensed under a Creative Commons Attribution-NonCommercial 4.0 International License.

\section{INTRODUCTION}

Aluminium phosphide (AlP) is a solid fumigant that is used to control rodents and pests in grain storage facilities in India. AlP poisoning has been found to be the most common cause of suicidal death in North India. AlP poisoning mortality rates vary from $40 \%$ to $80 \%$ and if the refractory myocardial depression develops then the mortality rate further increases even up to $100 \%$. When AlP comes into contact with water, it releases large quantities of phosphine (PH3), a very toxic gas and a mitochondrial poison. $\mathrm{PH}$ 3 inhibits mitochondrial cytochrome c oxidase thereby inhibiting cellular $\mathrm{O}_{2}$ utilization, inducing cellular damage by lipid peroxidation, direct toxic effect on cardiac myocytes, and adrenal gland with circulatory collapse. AlP has no effective antidote. Poisoning signs and symptoms include nausea, vomiting, restlessness, abdominal pain, palpitation, refractory shock, cardiac arrhythmias, pulmonary oedema, dyspnea, cyanosis, and sensory alterations. Diagnosis is based on clinical suspicion, positive silver nitrate paper test to phosphine, and gastric aspirate and viscera biochemistry. ${ }^{1-4}$

Veno-arterial extracorporeal membrane oxygenation (VA ECMO) can be a definitive therapy for AlP poisoning but should be considered for high-risk group as there is always risk of ECMO related complications.

\section{CASE REPORT}

Nineteen years old, previously healthy male came to our emergency room (ER) with alleged history of ingestion 
of five tablets of celphos poison (AlP). Patient arrived within $2 \mathrm{~h}$ of ingestion of poison. On arrival in ER, patient systolic blood pressure was less than $90 \mathrm{mmHg}$ was taken on ionotropic support, his blood gas suggestive of severe lactic metabolic acidosis $(\mathrm{pH} \leq 7.2$ initially, then worsened to 6.9) with lactate of 21, ECG showing wide complex arrhythmias and 2D Echocardiography suggestive of severe systolic dysfunction (left ventricular ejection fraction $<25 \%$ ).

Diagnosis was made of AlP poisoning with severe myocarditis and cardiogenic shock. Case management included resuscitation started with coconut oil through Ryles tube, intralipid injections, magnesium sulphate, soda bicarbonate and ionotropic support. After initial stabilisation and assessing the cardiac status of patient, we planned for VA ECMO. ECMO team was activated and patient shifted to the operation room. Patient was induced with injection ketamine $50 \mathrm{mg}$, fentanyl $100 \mathrm{ugm}$ and rocuronium $50 \mathrm{mg}$ intravenously. Patient is planned for VA ECMO with the femoral route. Left-sided femoral artery was cannulated with $18 \mathrm{~F}$ and distal perfusion canula. The right-sided femoral vein was cannulated with 24F cannula. After priming of ECMO circuit, patient was connected with circuit and put on ECMO support. Patient was shifted to the intensive care unit for further care and put on ventilator support. Renal replacement therapy was initiated with CRRT (CVVHDF) in view of severe acidosis. Monitoring was started as per our institutional protocol. Patient was weaned off from ECMO after $56 \mathrm{~h}$ successfully and weaned from ventilatory support in the next $24 \mathrm{~h}$ and discharged to the ward on the $10^{\text {th }}$ day of admission.

\section{DISCUSSION}

AlP is a solid fumigant that is used to control rodents and pests in grain storage facilities in India. AlP poisoning has been found to be the most common cause of suicidal death in North India. AlP poisoning mortality rates vary from $40 \%$ to $80 \%$ and if the refractory myocardial depression develops then the mortality rate further increases even up to $100 \%$. When AlP comes into contact with water, it releases large quantities of phosphine (PH3), a very toxic gas and a mitochondrial poison. Phosphine inhibits mitochondrial cytochrome c oxidase thereby inhibiting cellular $\mathrm{O}_{2}$ utilization, inducing cellular damage by lipid peroxidation, direct toxic effect on cardiac myocytes, and adrenal gland with circulatory collapse. AlP that can easily be bought and has no effective antidote. Poisoning signs and symptoms include nausea, vomiting, restlessness, abdominal pain, palpitation, refractory shock, cardiac arrhythmias, pulmonary oedema, dyspnea, cyanosis, and sensory alterations. Diagnosis is based on clinical suspicion, positive silver nitrate paper test to phosphine, and gastric aspirate and viscera biochemistry. ${ }^{1-4}$

AlP on exposure to hydrochloric acid and water in the stomach releases phosphine gas, which is rapidly absorbed gastrointestinal tract. Phosphine gas causes the release of free radicals and the inhibition of metabolic enzymes such as mitochondrial cytochrome $\mathrm{C}$ oxidase and causes cytotoxicity. Other pathognomic mechanisms are generation of highly reactive hydroxyl radicals due to the interaction between phosphine and hydrogen peroxide, and inhibition of catalase and peroxidase enzymes by phosphine resulting in lipid peroxidation due to the production of reactive hydroxyl radicals. Excessive oxidative stress causes an increase in glutathione reduction which results in cellular injury. Myocarditis is caused by the direct injury of cardiac myocytes by the toxic effects of phosphine gas leading onto cardiogenic shock.

There is no specific antidote for AlP poisoning. Conventional treatment methods, being used in our institutional settings are coconut oil through Ryle tube, intralipid injections, membrane stabilising agents such as magnesium sulphate, measures to control acidosis and ionotropic support as and when required. We had used conventional methods in the present case also. We consider VA ECMO for this case in view of worsening of cardiac status refractory to conventional treatment. Our case is in accordance with a study done by Mohan et al., ${ }^{4}$ in their study they concluded the significant role of VA ECMO in short term survival in AlP poisoning, however, they had only 15 patients in their ECMO group.

\section{CONCLUSION}

ECMO, complex in nature and carries its own risk, complications and expensive technology which should be reserved for patients with reversible disease process having high risk of mortality and not responding to conventional medical therapies. VA ECMO can be a definitive therapy for AlP poisoning which should be considered for highrisk group as there is always risk of ECMO related complications other than the AlP toxicity itself, thus further compromising the patient.

\section{REFERENCES}

1. Siwach SB and Gupta A. The profile of acute poisonings in Harayana-Rohtak study. J Assoc Physicians India. 1995;43(11):756-759.

2. Chugh SN, Dushyant, Ram S, Arora B and Malhotra KC. Incidence and outcome of aluminium phosphide poisoning in a hospital study. Indian J Med Res. 1991;94:232-235. 
3. Bogle RG, Theron P, Brooks P, Dargan PI, Redhead J. Aluminium phosphide poisoning. Emerg Med J. 2006;23(1):e3.

https://doi.org/10.1136/emj.2004.015941

4. Mohan B, Singh B, Gupta V, Ralhan S, Gupta D, Puri S, et al. Outcome of patients supported by extracorporeal membrane oxygenation for aluminum phosphide poisoning: An observational study. Indian Heart J. 2016;68(3):295-301.

https://doi.org/10.1016/j.ihj.2016.03.024

\section{Authors Contribution:}

AK- Concept and planning of case report; JP- Prepared first draft of manuscript; GA- Reviewed the case report; AKY- Revision of manuscript; A- Collection of details and revision of manuscript.

\section{Work attributed to:}

Kainos Super-Speciality Hospital, Rohtak - 124 001, Haryana, India.

\section{Orcid ID:}

Dr. Arvind Kumar-D https://orcid.org/0000-0001-9382-2588

Dr. Jai Prakash-io https://orcid.org/0000-0002-4884-5374

Dr. Gaurav Arya- iD https://orcid.org/0000-0002-5353-1089

Dr. Anand Kumar Yadav-10 https://orcid.org/0000-0003-3741-1834

Dr. Aman Dhankhar-(D) https://orcid.org/0000-0002-6101-0339

Source of Support: None, Conflict of Interest: None. 